\title{
Electrical stimulation disrupts biofilms in a human wound model and reveals the potential for monitoring treatment response with volatile biomarkers
}

Dol:

10.1111/wrr.12679

\section{Document Version \\ Accepted author manuscript}

Link to publication record in Manchester Research Explorer

Citation for published version (APA):

Ashrafi, M., Novak-frazer, L., Morris, J., Baguneid, M., Rautemaa-richardson, R., \& Bayat, A. (2018). Electrical stimulation disrupts biofilms in a human wound model and reveals the potential for monitoring treatment response with volatile biomarkers: ES disrupts biofilm and VOC allows monitoring. Wound Repair and Regeneration. https://doi.org/10.1111/wrr.12679

Published in:

Wound Repair and Regeneration

\section{Citing this paper}

Please note that where the full-text provided on Manchester Research Explorer is the Author Accepted Manuscript or Proof version this may differ from the final Published version. If citing, it is advised that you check and use the publisher's definitive version.

\section{General rights}

Copyright and moral rights for the publications made accessible in the Research Explorer are retained by the authors and/or other copyright owners and it is a condition of accessing publications that users recognise and abide by the legal requirements associated with these rights.

\section{Takedown policy}

If you believe that this document breaches copyright please refer to the University of Manchester's Takedown Procedures [http://man.ac.uk/04Y6Bo] or contact uml.scholarlycommunications@manchester.ac.uk providing relevant details, so we can investigate your claim.

\section{OPEN ACCESS}




\title{
Electrical stimulation disrupts biofilms in a human wound model and
} reveals the potential for monitoring treatment response with volatile

\section{biomarkers}

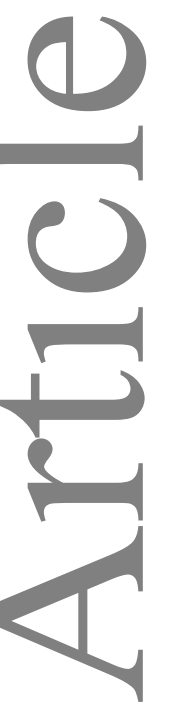

\author{
Mohammed Ashrafi MBChB ${ }^{1-3}$, Lilyann Novak-Frazer $\mathrm{PhD}^{2,4}$, Julie Morris ${ }^{5}$, Mohamed \\ Baguneid $\mathrm{MD}^{2}$, Riina Rautemaa-Richardson $\mathrm{PhD}^{2,4}$, Ardeshir Bayat MBBS, $\mathrm{PhD}^{1-2}$
}

${ }^{1}$ Plastic \& Reconstructive Surgery Research, Division of Musculoskeletal \& Dermatological Sciences, School of Biological Sciences, University of Manchester, Manchester, UK

${ }^{2}$ Manchester University NHS Foundation Trust, Wythenshawe Hospital, Manchester, UK

${ }^{3}$ Bioengineering Group, School of Materials, University of Manchester, Manchester, UK

${ }^{4}$ Manchester Academic Health Science Centre, Division of Infection, Immunity and Respiratory Medicine, School of Biological Sciences, Faculty of Biology, Medicine and Health, Manchester Academic Health Science Centre, The University of Manchester and Manchester University NHS Foundation Trust, Manchester UK

${ }^{5}$ Head of Medical Statistics, Honorary Reader in Medical Statistics, Manchester University NHS Foundation Trust, Wythenshawe Hospital, Manchester, UK

\section{Corresponding author:}

Dr Ardeshir Bayat, MBBS, PhD, Associate Professor, Plastic \& Reconstructive Surgery Research, Division of Musculoskeletal \& Dermatological Sciences, School of Biological Sciences, University of Manchester, Stopford Building, Oxford Road, Manchester M13 9PT, England, United Kingdom. Tel: 0161306 0607; Email: ardeshir.bayat@manchester.ac.uk

Short title: ES disrupts biofilm and VOC allows monitoring

Keywords: Biofilm, human, wound models, volatile organic compound, electrical stimulation

This article has been accepted for publication and undergone full peer review but has not been through the copyediting, typesetting, pagination and proofreading process, which may lead to differences between this version and the Version of Record. Please cite this article as doi: 10.1002/wrr.12679 


\begin{abstract}
Management of biofilm infections relies on time-consuming laboratory techniques and monitoring treatment by subjective clinical evaluations. Due to these limitations, there is a need to explore alternative strategies. The aims of this study were to assess the feasibility of using volatile organic compound (VOC) biomarkers to monitor treatment response and measure anti-biofilm efficacy of electrical stimulation (ES) in vitro and in human cutaneous wound biofilm models. Staphylococcus aureus (MSSA) and Pseudomonas aeruginosa (PA) biofilms were exposed to ES, ciprofloxacin, or both, with efficacy assessed and quantified by fluorescence staining, enumeration, metabolic assays and biomass quantification; VOCs were measured by gas chromatography-mass spectrometry. In vitro MSSA and PA and ex vivo PA biofilms exposed to ES showed significantly reduced bacterial viability, metabolic activity and biomass compared to controls $(\mathrm{P}<0.05)$. There was significant variation in the relative abundance of VOCs in in vitro MSSA and PA and in ex vivo PA biofilms exposed to ES and antibiotic $(\mathrm{P}<0.05)$. 2-methyl-1-propanol was associated with MSSA viability $(\mathrm{R}=0.93$, $\mathrm{P}<0.05)$, biomass $(\mathrm{R}=0.97, \mathrm{P}<0.05)$ and metabolic activity $(\mathrm{R}=0.93, \mathrm{P}<0.05)$ and 3-methyl-1butanol was associated with PA biomass $(\mathrm{R}=0.93, \mathrm{P}<0.05)$. We showed that $\mathrm{ES}$ and VOC biomarkers are possible options for alternative non-pharmacological anti-microbial management of biofilms and non-invasive monitoring of wound infection treatment responses, respectively.
\end{abstract}




\section{Introduction}

Biofilms are major contributors to delayed cutaneous wound healing, constituting $60 \%$ of chronic wounds and up to $80 \%$ of surgical site infections (SSIs) (1,2). Current anti-biofilm management relies heavily on antibiotics (3); however, due to the occurrence and spread of antibiotic-resistant bacteria, there is a growing need for alternative treatment methods (4). Also of concern is monitoring the treatment response which currently relies on subjective clinical evaluations, and objective laboratory techniques, such as culturing and sequencing. Clinical evaluation is heavily influenced by clinician variability and laboratory techniques are time-consuming and resource-dependent $(5,6)$. Therefore, there is an urgent need to investigate the monitoring and treatment of wound biofilms. A significant limiting factor is the availability of appropriate models of chronic wound biofilm infections using skin (7).

There is accumulating research in the use of volatile organic compounds (VOCs) to diagnose diseases and infections $(8,9)$. However, their use as biomarkers in monitoring disease progression and therapy efficacy is limited mainly to respiratory diseases and infections (10). The use of electrical stimulation (ES) to treat chronic wounds is currently utilised in clinical wound care management (11-13) and recent reviews have clearly highlighted its beneficial effects $(14,15)$. Although the antimicrobial effects of ES are well-documented in vitro (16) and animal studies (17), its efficacy against cutaneous wound biofilms is sparse (18). The porcine model is the most common ex vivo skin/wound substrate used to study biofilm formation (19-23). Anatomically and physiologically, pig skin is similar to human skin (24), however it is not identical, leading to possible discrepancies when translating findings into clinical practice. Therefore it is necessary to develop human skin models to emulate clinical conditions to allow meaningful interpretations of experimental findings (25). 
Therefore, the threefold aims of this study were to assess the feasibility of using VOC biomarkers to monitor treatment response against cutaneous wound biofilms; to assess the anti-microbial efficacy of ES in a clinically relevant human cutaneous wound biofilm model; and finally, to use this model to validate the use of VOCs in assessing wound infections. 


\section{Materials and Methods}

\section{Study design}

Bacterial biofilms were grown on plastic coverslips and excisional human cutaneous wound tissue explants in broth medium at $37^{\circ} \mathrm{C}$ for 1 or 3 days. Pre-grown biofilms were exposed to ES, antibiotics or ES followed by antibiotic exposure. Five methods were used to evaluate the effects of treatments on biofilms. Biofilm visualisation was by wide-field, bright-field and fluorescence microscopy of multiply-stained sections. Biofilm viability was determined quantitatively by enumeration and fluorescent live/dead staining. The XTT cell proliferation assay was used to determine biofilm metabolism and the amount of double stranded DNA reflected biofilm biomass (26). VOCs were identified using gas chromatography-mass spectrometry (GCMS). All in vitro experiments were done twice in triplicate and all ex vivo experiments were done four times in triplicates (Figure 1).

\section{Bacterial strains}

Bacterial isolates were American Type Culture Collection reference strains obtained from LGC Standards (Teddington, UK). The bacterial strains used in this study were Staphylococcus aureus ATCC 29213 (MSSA) which is a methicillin-sensitive wound isolate and Pseudomonas aeruginosa ATCC 27316 (PA) which is a human wound isolate. All strain stocks were frozen and stored long term at $-80^{\circ} \mathrm{C}$. All isolates were revived from frozen stocks onto Columbia agar containing horse blood (E\&O Laboratories Ltd, Bonnybridge, Scotland) and incubated at $37^{\circ} \mathrm{C}$ for $16-18 \mathrm{~h}$ prior to use. 


\section{Ex vivo cutaneous excisional wound organ culture models}

Full thickness cutaneous tissue was obtained from human patients $(n=2 ; 2$ female; mean age 40 years) undergoing elective surgery ( 1 abdominoplasty; 1 breast reduction) with appropriate ethical approval. The skin underwent intra-operative disinfection with $0.5 \%$ (v/v) aqueous chlorhexidine. Tissue was trimmed of excess adipose tissue and disinfected with $2 \%(\mathrm{v} / \mathrm{v})$ chlorhexidine gluconate and 70\% (v/v) isopropyl alcohol (ChloraPrep ${ }^{\mathrm{TM}}$ Sepp $^{\mathrm{TM}}$ applicator, $\mathrm{BD}$, Basingstoke, UK) by rubbing the skin surface gently for 30 seconds. A sample of disinfected tissue was cultured both aerobically and anaerobically for 24 hours onto Columbia agar containing horse blood to confirm elimination of normal skin flora. Eight $\mathrm{mm}$ circular biopsies were taken from the main tissue sample using disposable biopsy punches (Integra ${ }^{\mathrm{TM}}$ Miltex $^{\circledR}$, York, PA, USA). In the centre of these circular skin explants, a $3 \mathrm{~mm}$ diameter circular excisional artificial wound was created to a depth of $2 \mathrm{~mm}$ by a further punch biopsy. Explants were submerged into sterile 24-well Corning ${ }^{\circledR}$ Costar® cell culture plates (SigmaAldrich, Poole, UK), with each well containing $0.5 \mathrm{ml}$ serum-free Williams E culture medium (ThermoFisher Scientific ${ }^{\mathrm{TM}}$, Basingstoke, UK) supplemented with 1\% (v/v) non-essential amino acid solution, $10 \mu \mathrm{l} / \mathrm{ml}$ insulin, $10 \mathrm{ng} / \mathrm{ml}$ hydrocortisone and $2 \mathrm{mmol}$ L-glutamine (27). Explants were maintained at $37^{\circ} \mathrm{C}$ in a stationary carbon dioxide $(5 \%)$ incubator and were cultured for a minimum of 24 hours with daily medium change prior to use and were not utilised beyond 7 days to maintain explant viability (28).

\section{Biofilm formation on an in vitro cell culture model}

To generate a fresh supply of cells, a $50 \mathrm{ml}$ centrifuge tube containing $25 \mathrm{ml}$ Mueller Hinton Broth (supplier) was inoculated with a single colony of MSSA or PA and incubated at $37^{\circ} \mathrm{C}$ in a shaking incubator at $250 \mathrm{rpm}$ overnight. Following this, a $50 \mathrm{ml}$ centrifuge tube containing 
$25 \mathrm{ml}$ Mueller Hinton Broth was inoculated with $200 \mu \mathrm{L}$ of the overnight culture and incubated at $37^{\circ} \mathrm{C}$ in a shaking incubator at $250 \mathrm{rpm}$ until the culture reached an optical density between 0.1 and 0.2 . A 13 mm diameter Nunc ${ }^{\mathrm{TM}}$ Thermanox ${ }^{\mathrm{TM}}$ Coverslip (Thermo Scientific, Hampshire, UK) was used as the inert substrate for biofilm formation. Coverslips was placed with the treated surface facing upwards into a sterile 24 well Corning ${ }^{\circledR} \operatorname{Costar}{ }^{\circledR}$ cell culture plate and covered with $1 \mathrm{ml}$ of MSSA or PA bacterial suspension. The plates were then incubated at $37^{\circ} \mathrm{C}$ in a stationary carbon dioxide (5\%) incubator for up to 1 or 3 days. Appropriate controls inoculated with $1 \mathrm{ml}$ sterile Mueller Hinton broth were also prepared and incubated for up to 1 or 3 days.

\section{Biofilm formation on ex vivo cutaneous wound organ culture models}

PA bacterial suspensions for ex vivo explants were prepared in the same way as for in vitro biofilms, as described above. An $8 \mathrm{~mm}$ excisional cutaneous wound explant was used as the substrate for biofilm formation. The explant was placed with the wounded surface facing up into a sterile 24 well Corning ${ }^{\circledR}$ Costar ${ }^{\circledR}$ cell culture plate. PA bacterial suspension $(1 \mathrm{ml}$ at 0.1-0.2 OD) was pipetted into the well submerging the wound explant. The plates were then incubated at $37^{\circ} \mathrm{C}$ in a stationary carbon dioxide (5\%) incubator for up to 3 days. Samples of inoculated tissue were cultured aerobically for 24 hours on Columbia agar containing horse blood to confirm mono-microbial biofilm formation at 3 days. Appropriate controls inoculated with $1 \mathrm{ml}$ sterile Mueller Hinton broth were also prepared and incubated up to 3 days. 


\section{Biofilm exposure to ES and antibiotics}

\section{$\underline{\text { In vitro biofilms, days } 1 \text { and } 3}$}

MSSA and PA biofilms were subjected to the following:

$E S$ - Biofilms were subjected to direct current (DC) ES waveform for 30 minutes at an electric field of $100 \mathrm{mV} / \mathrm{mm}(28,29)$. This electric field corresponds to the endogenous "current of injury" prevailing in cutaneous wound healing during initiation of granulation tissue, fibroplasia, wound contraction and neovascularisation $(30,31)$. To deliver DC to the biofilm, coverslips were placed in the middle of a glass petri dish (radius: $25 \mathrm{~mm}$ ) containing $2.5 \mathrm{ml}$ Mueller Hinton broth. The current was received directly by the $\mathrm{Ag}-\mathrm{AgCl}$ electrodes, which were dipped in Steinberg's saline solution $(60 \mathrm{mM} \mathrm{NaCl}, 0.7 \mathrm{mM} \mathrm{KCl}, 0.8 \mathrm{mM}$ $\mathrm{MgSO}_{4} .7 \mathrm{H}_{2} \mathrm{O}, 0.3 \mathrm{mM} \mathrm{CaNO}_{3} .4 \mathrm{H}_{2} \mathrm{O}, 1.4 \mathrm{mM}$ Tris base maintained at $\mathrm{pH}$ 7.4). The inverted "U" shaped agar bridges filled with Steinberg's saline solution and gelled with 1\% (w/v) agar (A/1080/48, Fisher Scientific, Loughborough, UK) carried the current from the solution into the petri dish. This set up for DC was developed in order to prevent the formation of air bubbles that would be generated if the electrodes were placed directly into the Petri dish. The power supply (TTi EX752M) was set to constant current and the voltage adjusted until the Petri dish reached the desired field strength. A multimeter (TENMA model 72-7745, Ohio, USA) was used to calculate voltage between the ends of the electrodes inside the ES chamber. This was performed every 15 minutes and was maintained consistently at $100 \pm 3 \mathrm{mV} / \mathrm{mm}$ for the total duration of ES (30 minutes). Coverslips were then incubated in Mueller Hinton broth at $37^{\circ} \mathrm{C}$ in a stationary carbon dioxide $(5 \%)$ incubator for 24 hours.

Antibiotic - Biofilms were subjected to ES using the same setup without any stimulation and then incubated in ciprofloxacin- $\mathrm{HCl}$ at $37^{\circ} \mathrm{C}$ in a stationary carbon dioxide (5\%) incubator for 
$24 \mathrm{~h}$. A concentration of $10000 \mathrm{mg} / \mathrm{L}$ was chosen, as preliminary work identified this concentration of ciprofloxacin resulted in less than $10 \%$ biofilm viability irrespective of species or time point (Figure S1).

Controls or non-treated - Biofilms were subjected to ES using the same setup without any stimulation and then incubated in Mueller Hinton broth at $37^{\circ} \mathrm{C}$ in a stationary carbon dioxide $(5 \%)$ incubator for $24 \mathrm{~h}$.

\section{$\underline{\text { Ex vivo biofilms, day } 3}$}

PA biofilms were subjected to the following:

$E S$ - Biofilms were subjected to DC ES waveform for 30 minutes at an electric field of 100 $\mathrm{mV} / \mathrm{mm}$ as described above and were then incubated in Mueller Hinton broth at $37^{\circ} \mathrm{C}$ in a stationary carbon dioxide (5\%) incubator for 24 hours.

Antibiotic - Biofilms were subjected to ES using the same setup without any stimulation and then incubated in ciprofloxacin at $37^{\circ} \mathrm{C}$ in a stationary carbon dioxide $(5 \%)$ incubator for 24 hours. A concentration of $20 \mathrm{mg} / \mathrm{L}$ was chosen based on work conducted by Brunner et al (32) which identified a single dose of $400 \mathrm{mg}$ ciprofloxacin given intravenously led to a concentration of approximately $20 \mathrm{mg} / \mathrm{L}$ over a 24 hour period at a cantharis-induced blister site in healthy individuals. 
ES + antibiotic - Biofilms were subjected to DC ES waveform for 30 minutes at an electric field of $100 \mathrm{mV} / \mathrm{mm}$ ES followed by incubation in ciprofloxacin $(20 \mathrm{mg} / \mathrm{L})$ at $37^{\circ} \mathrm{C}$ in a stationary carbon dioxide (5\%) incubator for 24 hours.

Controls or non-treated - Biofilms were subjected to the same setup as for ES without any stimulation and then incubated in Mueller Hinton broth at $37^{\circ} \mathrm{C}$ in a stationary carbon dioxide (5\%) incubator for 24 hours.

\section{Biofilm visualisation}

Wound tissue explants were embedded in optical cutting temperature (OCT) compound (KPCryoCompound, Klinipath, Duiven, Netherlands) post treatment, then snap-frozen in liquid nitrogen and stored at $-80^{\circ} \mathrm{C}$ before cryosectioning. Ten micrometer sections of were prepared with a cryostat microtome (OTF5000, Bright Instruments Ltd, UK). Each section was fixed in cold acetone for 10 minutes, which was then allowed to evaporate at room temperature. Slides were immersed in sterilised PBS to remove residual OCT.

\section{$\underline{\text { Live/Dead staining }}$}

Coverslips were stained post-exposure with FilmTracer ${ }^{\mathrm{TM}}$ LIVE/DEAD ${ }^{\circledR}$ Biofilm Viability Kit (Invitrogen Molecular Probes ${ }^{\circledR}, \mathrm{NY}, \mathrm{USA}$ ) as per the manufacturer's instructions to visualise the effects of ES and/or antibiotics on viability and architecture. Images were collected on a Zeiss Axioimager.M2 upright microscope using a 40x/1.3 EC Plan-neofluar objective and captured using a Coolsnap HQ2 camera (Photometrics) through Micromanager 
software v1.4.23. Specific band pass filter sets for FITC and Texas Red were used to prevent bleed between channels. Images were then processed and analysed using Fiji ImageJ (http://imagej.net/Fiji/Downloads).

\section{Haematoxylin and Eosin staining}

Tissue sections were stained for nuclei post-exposure with haematoxylin (Sigma-Aldrich, Saint Louis, USA) and cytoplasm was counterstained with eosin (Sigma-Aldrich, Saint Louis, USA) in a Varistain ${ }^{\mathrm{TM}}$ 24-4 Automatic Slide Stainer (ThermoFisher Scientific, Basingstoke, UK). This involved fixation of sections for 2 minutes in $70 \%(\mathrm{v} / \mathrm{v})$ industrial methylated spirits (IMS), followed by a 2 minute wash in water. Sections were stained for 2 minutes in haematoxylin Gills 2, and then in water. Tissue sections were then washed in 5\% (v/v) acetic acid and dehydrated in graded IMS/ethanol (70\%, 90\% and 100\% (v/v)). Sections were counterstained with alcoholic eosin $\mathrm{Y}$ solution for 90 seconds followed by three $100 \%(\mathrm{v} / \mathrm{v})$ ethanol washes. Finally, slides were clarified with xylene and then mounted in mounting media. Images were collected on an Olympus BX63 upright microscope using a 60x/1.42 PlanApo N and 100x/1.40 Uplan SApo objectives and captured and white-balanced using a DP80 camera (Olympus) in colour mode through CellSens Dimension v1.16 (Olympus). Images were then processed and analysed using Fiji ImageJ (http://imagej.net/Fiji/Downloads).

\section{Gram-Twort staining}

Tissue sections were stained post-exposure with Gram-Twort for the demonstration of bacteria in tissue sections. Sections were brought down to water and stained in crystal violet 
solution followed by Gram's iodine for 3 minutes each. Tissue sections were then dried at $60^{\circ} \mathrm{C}$ and then differentiated in $2 \%(\mathrm{v} / \mathrm{v})$ acetic acid alcohol for 13 minutes at $56^{\circ} \mathrm{C}$. Sections were counterstained with Twort's stain for 5 minutes followed by dehydration in ethanol. Finally, slides were clarified with xylene and then mounted in mounting media. Images were collected on an Olympus BX63 upright microscope as described above.

\section{$\underline{\text { DAPI + Concanavalin A staining }}$}

Tissue sections were stained post-exposure with Concanavalin-A (Con-A) fluorescent stain and then incubated overnight at $4^{\circ} \mathrm{C}$ in the dark. The sections were then rinsed gently with PBS to remove excess stain. Sections were counterstained with 4',6-diamidino-2-phenylindole (DAPI) by incubating for a further 15 minutes at room temperature in the dark. Sections were finally rinsed gently with PBS. Images were collected on a Zeiss Axioimager.M2 upright microscope as described above.

\section{Peptide nucleic acid-fluorescence in situ hybridisation (PNA-FISH) staining}

Tissue sections were stained post-exposure with a drop of a PNA-FISH-TexasRed conjugated to a specific universal bacterial (UniBac) 16S ribosomal RNA probe (AdvanDx, Woburn, MA, USA). Samples were incubated for $90 \mathrm{~min}$ at $55^{\circ} \mathrm{C}$. The coverslip was removed, and the slides were washed in warm washing buffer at $55^{\circ} \mathrm{C}$ (AdvanDx, USA) for 30 min and then air dried in the dark. A drop of mounting medium was placed on top of the slide, which was then covered with a coverslip and air dried for $15 \mathrm{~min}$. Images were collected on a Zeiss Axioimager.M2 upright microscope as described above. 


\section{Biofilm viability}

\section{$\underline{\text { Enumeration }}$}

Coverslips and wound tissue explants post-exposure were submerged in $1 \mathrm{ml}$ PBS and placed in an ultrasonic bath at $60 \mathrm{~Hz}$ and $60 \mathrm{~W}$ for 5 minutes with 30 seconds of vortexing either side to remove the remnants of the adherent biofilm. CFU assessment of the resultant bacteria was done in triplicate onto agar medium. These values were subsequently log transformed prior to statistical analyses.

\section{$\underline{\text { Live/Dead staining quantification }}$}

Definiens Tissue Studio software version 3.5.1 (Definiens, Munich, Germany) was used for analysis of LIVE DEAD $^{\circledR}$-stained coverslips post-exposure and presented as viable bacteria $/ \mathrm{mm}^{2}$. These values were subsequently log-transformed prior to statistical analyses.

\section{Biofilm metabolic activity}

\section{$\underline{\text { Substrate in situ }}$}

The metabolic activity of biofilms grown on coverslips post-exposure was assessed using the XTT cell proliferation assay (33). A saturated solution was prepared from XTT reagent (Sigma-Aldrich, Poole, UK) in 1 X PBS at a concentration of $0.5 \mathrm{~g} / \mathrm{L}$. To activate XTT, $100 \mu \mathrm{l}$ $1 \mathrm{mM}$ menadione (Sigma-Aldrich, Saint Louis, USA) was added to every $10 \mathrm{ml}$ XTT solution used. Activated XTT $(400 \mu \mathrm{l})$ was added to each well, incubated for 2 hours at $37^{\circ} \mathrm{C}$ in the dark and the colour change measured using a POLARStar Omega spectrophotometer (BMG Labtech, Ortenberg, Germany) at an absorbance of 490 and $630 \mathrm{~nm}$. Results were processed 
using Omega software (BMG Labtech, Ortenberg, Germany) and $630 \mathrm{mn}$ absorbance values subtracted from the $490 \mathrm{~nm}$.

\section{Biofilms detached from substrate}

The metabolic activity of biofilms grown on coverslips and wound tissue explants, then detached post-exposure, were also assessed using the XTT cell proliferation assay. To separate biofilms, coverslips or explants were submerged in $1 \mathrm{ml}$ PBS and placed in an ultrasonic bath at $60 \mathrm{~Hz}$ and $60 \mathrm{~W}$ for 5 minutes with 30 seconds of vortexing pre- and postsonication. Biofilm material was sedimented by centrifugation at $12000 \mathrm{~g}$ for 5 minutes. A saturated solution of XTT was prepared and activated as described above. PBS was removed and $400 \mu \mathrm{l} /$ well activated XTT solution was added. Samples were vortexed for 30 seconds to re-suspend the biofilm and subsequently processed as described above.

\section{Biofilm biomass}

Biofilm dsDNA, representing biomass, was extracted by separating the biofilm from its respective substrate and measured using the fluorescent nucleic acid Quant-iT PicoGreen dsDNA reagent (Molecular Probes Inc., USA). Nucleic acids were extracted from biofilms using the QIAamp DNA Mini Kit (QIAGEN, Valencia, CA), according to the manufacturer's recommendations. Nucleic acid extracts and the PicoGreen reagent were mixed thoroughly before fluorometric analysis at $492 \mathrm{~nm}$ (BMG Labtech, UK). Lambda DNA included in the kit was used to construct the standard curve (concentration range $0-1000 \mathrm{ng} / \mathrm{ml}$ ) according to the manufacturer's instructions. 


\section{Gas chromatography-mass spectrometry (GCMS)}

Precision thread headspace vials (20 ml, Fisher Scientific, Loughborough, UK) sealed with universal magnetic screw caps with PTFE/butyl penetrable septa (Fisher Scientific, Loughborough, UK) were used as they were shown to be optimum for the containment of VOCs for headspace sampling and GCMS analysis.

GCMS analyses were performed using an Agilent 5977A MSD mass spectrometer (Agilent Technologies, Santa Clara, CA, USA) linked to GC (Agilent 7890B GC system, Agilent Technologies, Santa Clara, CA, USA) which was equipped with an autosampler (PAL RSI 85 autosampler system, Agilent Technologies, Santa Clara, CA, USA). Post-exposure coverslips and wound tissue explants were sealed in headspace vials for 24 hours at $37^{\circ} \mathrm{C}$ prior to headspace sampling which consisted of agitating at $37^{\circ} \mathrm{C}$ for 5 minutes. A headspace gas sample $(1 \mathrm{ml})$ was collected using an Agilent gas-tight syringe (Agilent Technologies, Santa Clara, CA, USA) attached to the autosampler. This was injected directly onto a DB-Wax column (30 m, $0.32 \mathrm{~mm}$ inner diameter, $0.25 \mu \mathrm{m}$ film thickness (Agilent Technologies, Santa Clara, CA, USA)) through an injector (split-less) and separated using $1.02 \mathrm{ml} / \mathrm{min}$ column flow. The oven temperature profile was $40^{\circ} \mathrm{C}$ for 5 minutes, increased to $230^{\circ} \mathrm{C}\left(10^{\circ} \mathrm{C} / \mathrm{min}\right)$ over 19 minutes and finished with 5 minutes at $230^{\circ} \mathrm{C}$. VOCs were detected in the mass to charge $(\mathrm{m} / \mathrm{z})$ range of $25-350$ Da.

Chromatograms were processed with MassHunter qualitative analysis software (Agilent Technologies, Santa Clara, CA, USA) prior to further statistical analyses. Chromatograms were processed by introducing a 2 minute solvent delay, noise removal and by comparing each inoculated sample to its corresponding time point control. Peaks based on retention times 
unique to inoculated samples underwent baseline subtraction and the resulting mass spectra were tentatively identified based on forward and reverse match searches of the National Institute of Standards and Technology library. The area of each unique chromatographic peak was calculated to provide relative abundances. A peak common to all samples and controls was identified and the area of each unique chromatographic peak was normalised to this common peak to account for GCMS variations over the sampling duration. A value of 1 was added to all normalised peak areas and subsequently log transformed prior to statistical analyses.

\section{Statistical analysis}

Continuous data were summarised as the mean $\pm 95 \%$ confidence intervals. A generalised estimating equations (GEE) model with pairwise Bonferroni corrections was used for all the biofilm parameters measured: viability (colony-forming unit (CFU) assessment and live/dead quantification), metabolic activity (XTT assay), biomass (PICO green dsDNA assay) and relative abundances of VOCs. Spearman's correlation coefficient was calculated to assess the association between biofilm viability, metabolism or biomass and relative abundances of VOCs (34). A P value of $<0.05$ was considered statistically significant. Statistical analyses were performed using SPSS for Windows version 22.0 (SPSS, IBM, Armonk, NY, USA) and graphical representation using GraphPad Prism 7 (GraphPad Software, La Jolla, CA, USA).

\section{Ethics statement}

Written informed consent was obtained from patients for tissue samples. Permission was granted by NHS National Research Ethics Service. 


\section{Results}

\section{ES reduces viability of MSSA and PA biofilms in vitro}

Viability, as determined by enumeration and fluorescence quantification, was significantly reduced in methicillin sensitive Staphylococcus aureus (MSSA) biofilms exposed to ES at day 1 (P $<0.05$; Figures $2 \mathrm{~A}$ and B; Table $\mathrm{S} 1)$. Visualisation confirmed reduced day 1 MSSA viability, with biofilm disruption appearing as sparse patches of viable colonies (Figure 2F). MSSA biofilms matured by day 3 as shown by the increasing separation of areas of heavily concentrated viable bacteria (Figure $2 \mathrm{~F}$ ), with some highly dense areas suggesting the presence of foci of dissemination, typical of mature biofilms. Biofilms exposed to ES appeared with fewer concentrated nodules; however, viability was not significantly reduced ( $\mathrm{P}>0.05$; Figures $2 \mathrm{~A}$ and $\mathrm{B})$. There was a significant reduction in viability of Pseudomonas aeruginosa (PA) biofilms exposed to ES at day 1 and day $3(\mathrm{P}<0.05$; Figures $3 \mathrm{~A}$ and $\mathrm{B})$. This was confirmed visually with a reduction in viable cells (green stained) and a sparsity of colonies (Figure 3F).

\section{ES decreases metabolic activity and biomass of MSSA and PA biofilms in vitro}

The metabolic activity of biofilms was determined using the XTT reduction assay either with the coverslip in situ or with detached biofilms. Metabolic activity of MSSA biofilms exposed to ES was significantly reduced by day 3 when assessed in situ $(\mathrm{P}<0.05$; Figure $2 \mathrm{C})$. Moreover, metabolic activity was significantly reduced at day 1 and day 3 in these biofilms were detached from the substrate $(\mathrm{P}<0.05$; Figure 2D). ES-treated PA biofilms on days 1 and 3 showed significant reduced metabolic activity irrespective of the assay method used $(\mathrm{P}<$ 0.05; Figures 3C and D). The trends of metabolic activity reduction in ES exposed MSSA and 
PA biofilms was the same in both techniques. The removal of the biofilm from the substrate followed by XTT reduction assay may provide a more accurate representation of its metabolic activity, as the XTT compound is able to better penetrate through all areas of the biofilm which may not be possible in biofilms left attached to the substrate. Therefore, further metabolic activity quantification in the ex vivo model was conducted using this latter protocol. Biomass was significantly reduced in day 3 MSSA and PA biofilms exposed to ES ( $\mathrm{P}<0.05$; Figures $2 \mathrm{E}$ and $3 \mathrm{E}$ ). ES had no significant effect on the biomass of early (day 1) MSSA and PA biofilms.

ES effects PA biofilm viability, metabolic activity and biomass in a clinically relevant $e x$ vivo model

Viability, metabolic activity and biomass were significantly reduced in ex vivo PA biofilms exposed to ES, antibiotic and in combination (ES + Antibiotic) compared to untreated controls $(\mathrm{P}<0.05$; Figure 4$)$. PA biofilm viability $(\mathrm{P}<0.05)$, metabolic activity $(\mathrm{P}<0.05)$ and biomass $(\mathrm{P}<0.05)$ were all reduced significantly when exposed to antibiotics or antibiotics plus ES as compared to ES exposure alone.. Biofilm visualisation confirmed the above findings with biofilm thickness reduced following ES exposure compared to controls but with a greater reduction in biofilms exposed to antibiotic alone or in combination with ES (Figures 5A-E). This pattern of reduced biomass was confirmed visually with concanavalin A and in in situ fluorescence hybridisation (FISH)-stained sections (Figures 5E-F). Exposure to ES followed by antibiotic treatment had no significant impact on PA biofilm viability, metabolic activity or biomass when compared to antibiotic only treated biofilms $(\mathrm{P}>0.05)$. 


\section{VOC biomarker variations allow monitoring of response to treatment}

VOC profiling by GCMS of 108 MSSA and PA in vitro biofilms exposed to ES, antibiotics or no treatment was undertaken to identify potential biomarkers that could be used to monitor response to treatment. Butanedione and acetic acid ethenyl ester were only identified in MSSA biofilms exposed to ciprofloxacin $(\mathrm{P}<0.05$; Figures $6 \mathrm{~A}$ and $\mathrm{B})$. The relative abundances of 2-methyl-1-propanol and 3-methyl-1-butanol were significantly reduced in antibiotic-exposed MSSA biofilms on days 1 and 3 compared to those exposed to ES or untreated, control biofilms $(\mathrm{P}<0.05)$. The relative abundance of 2 -methyl-1-propanol was also significantly reduced in ES-exposed MSSA biofilms on day 3 compared to controls $(\mathrm{P}<$ $0.05)$.

The relative abundance of hydrogen cyanide, 5-methyl-2-hexanamine, 5-methyl-2heptanamine, 1-undecene, 3-methyl-1-butanol and 2-nonanone was significantly reduced in PA biofilms (on both days 1 and 3) exposed to antibiotic compared to those exposed to ES or untreated, control biofilms ( $\mathrm{P}<0.05$; Figures 6C and D). Hydrogen cyanide, 5-methyl-2hexanamine, 1-undecene, 3-methyl-1-butanol and 2-nonanone were significantly reduced in ES-exposed day 3 PA biofilms compared to untreated controls $(\mathrm{P}<0.05)$.

The application of VOC biomarkers in treatment response monitoring in a clinically relevant ex vivo model

VOC profiling by GCMS of 72 ex vivo PA biofilms exposed to ES, antibiotics, or in combination (ES + Antibiotic) revealed five VOCs unique to PA biofilms. The relative abundance of hydrogen cyanide, 5-methyl-2-hexanamine, 5-methyl-2-heptanamine and 1- 
undecene was significantly reduced in antibiotic-exposed PA biofilms alone or in combination with ES (P < 0.05; Figure 6E). Levels of 5-methyl-2-hexanamine, 5-methyl-2heptanamine, 1-undecene and 2-nonanone were significantly reduced in PA biofilms exposed to ES only $(\mathrm{P}<0.05)$.

VOC biomarkers in MSSA and PA biofilms correlated with viability, metabolic activity and biomass

MSSA production of 2-methyl-1-propanol correlated positively with biofilm viability $(\mathrm{R}=$ 0.93, $\mathrm{P}<0.05$ : Figures 7A and $\mathrm{B})$, biomass $(\mathrm{R}=0.97 ; \mathrm{P}<0.05$; Figure $7 \mathrm{C})$ and metabolic activity $(\mathrm{R}=0.93$; $\mathrm{P}<0.05$; Figure $7 \mathrm{D})$; meanwhile PA production of 3-methyl-1-butanol positively correlated with biofilm biomass $(\mathrm{R}=0.93$; $\mathrm{P}<0.05$; Figure $7 \mathrm{E})$. 


\section{Discussion}

Biofilms present in chronic wounds account for up to $60 \%$ of infections (1) and in SSIs, where the prevalence is even higher (2). Biofilms cause chronic infections due to increased tolerance to antibiotics and resistance against the body's immune system (35). Therefore, this study attempted to address this clinical concern by determining whether ES could be useful in the management of wound biofilms. Endogenous direct current (DC) electric fields are fundamental components of development and regeneration, which suggest a critical role in wound healing (36). DC, a monophasic electrical waveform, was used to successfully reduce MSSA and PA biofilm viability, metabolic activity and biomass in vitro. Its application to mature PA biofilms in a human ex vivo excisional cutaneous wound model confirmed a reduction in all these parameters compared to untreated controls. We identified that ES reduced biomass; this corroborates previous findings which found ES reduced biofilm attachment and adherence, key factors in biofilm formation and development (37). While the use of ES in wound care management is well established (38), we did not identify any previous studies assessing the impact of ES on wound biofilms in a human ex vivo model. Investigations of ES against biofilms in vivo are also sparse, with only six studies to date having assessed the effect of ES on biofilms, with the majority showing positive outcomes (39-44); however, none of these studies investigated the direct effect of ES on cutaneous wound bacterial biofilms.

ES, however, was not as effective in reducing biofilm compared to ciprofloxacin in both in vitro and ex vivo models. The effectiveness of ciprofloxacin was not unexpected in vitro as the concentration used reduced biofilm metabolic activity by greater than ninety percent. This high dose was chosen as a positive control to allow effective comparisons between changes to relative abundances of VOCs in biofilms exposed to ciprofloxacin which were expected to 
have a significant anti-biofilm effect compared to clinically useable ES parameters, where the anti-biofilm effect was unknown. In the ex vivo model, despite the use of a clinically relatable concentration of ciprofloxacin being used (32); it was still more effective against PA biofilms compared to ES exposure. Moreover, only a single dose of either treatment was investigated. With the increasing concern of antibiotic resistance, prolonged or repeated ES treatment may have a role in anti-biofilm management. Further studies to determine if repeated or prolonged ES use could be as effective or could complement and perhaps even replace antibiotic treatment are necessary. Also, the use of ES as an alternative therapy choice may be more relevant against antibiotic-resistant bacterial strains; further experimental work, including resistant strains is necessary to clarify the circumstances where ES may be most beneficial.

ES may increase the efficacy of antibiotics, a concept termed the "bioelectric effect"; and this has been shown in the majority of studies to date which have used DC (45). Many have shown an enhancing effect of ES on the efficacy of antimicrobials in vitro $(46,47)$ although we were not able to demonstrate this in vitro or ex vivo in the present study. However, we only assessed the bioelectric effect against one species at a single time point with the use of a single antibiotic agent at a high, fixed concentration. Del Pozo et al proposed that the bioelectric effect is not universal across all microorganisms and antimicrobials and its use should probably be selective (48). Antibiotic-specific bioelectric effects have been shown previously (49) where certain antibiotics were enhanced with the addition of ES, whereas others had no increased efficacy against Pseudomonas biofilms. Variations in intensity of the currents can also enhance the bioelectric effect (46); however, caution must be applied as there will be limitations on the ES parameters deemed safe for use in the clinical setting. The applied electric field we used corresponds to the endogenous "current of injury" which is the lateral electrical field that is generated through the wound following an injury to the skin, 
thought to be significant in initiating repair (50) and deemed safe in the clinical environment $(30,31)$.

A major limitation when investigating wound biofilm diagnostic and treatment methods is the availability of appropriate skin wound biofilm models (7). The porcine ex vivo wound model is most commonly used for investigating biofilm formation and assessing therapeutics (19-22, 51). However, porcine skin is not identical to human skin, yet there are very few studies utilising human skin as a substrate for biofilm formation $(52,53)$. We have previously established the formation of common cutaneous wound pathogenic bacterial biofilms on a human ex vivo excisional cutaneous wound model (25). Here, we successfully used this human cutaneous biofilm wound model to assess the efficacy of ES against biofilms and to determine if it was possible to use VOC biomarkers in monitoring treatment response.

This study demonstrated the application of VOC biomarkers to monitor bacterial biofilm response to treatment with potential clinical use. Current monitoring of wound infection response to treatment involves a combination of clinical indicators which are subjective and microbial sampling for laboratory culturing and sequencing, which are limited by time and accuracy $(5,54)$. The use of VOCs and its application in point of care testing, such as electronic nose technologies in wound infection diagnoses, have been investigated and discussed previously $(55,56)$. As with the use of VOC profiles in the diagnosis of lung disease, their use as potential markers in monitoring treatment response have been more robustly investigated $(8,57)$, compared to their use in monitoring wound infection response to treatment, which is limited (58). Here we showed dynamic changes in VOC profiles in response to antibiotic- or ES-treated biofilms in vitro and confirmed these findings in our 
clinically relevant human ex vivo wound biofilm model. These results imply that detection of VOCs and their various profiles can be harnessed for point of care testing which would ameliorate wound infection diagnosis and treatment. Of interest was the detection of butanedione and acetic acid ethenyl ester in ciprofloxacin-treated MSSA biofilms and these two VOCs, could be breakdown products of MSSA biofilms. However, these findings are exploratory and further experimental work including multiple time points and bacterial strains are necessary before firm conclusions can be drawn about their usefulness.

This study has a number of limitations. Only two bacterial strains were used as mono-cultures in vitro and one in the ex vivo model, whilst most chronic wounds comprise poly-microbial biofilms (1), although single pathogen wound infections do occur. A natural progression of this work is to investigate the impact of ES and VOC detection in Gram positive and polymicrobial biofilms using our ex vivo model.. The ex vivo model developed more closely portrays a human skin wound than our or other published in vitro models; but direct translation of the results to the clinical setting should be met with caution. Although we found statistically significant reduction (greater than 60\%) in bacterial viability of biofilms exposed to ES, this is not a clinically significant reduction in terms of chronic wound healing, and therefore, human trials are necessary to validate our findings. While certain differences between controls and treatment modalities were statistically significant, the absolute reductions were small and may not be clinically significant. In addition to our previously published model (59), other methods of explant inoculation, such as surface and intradermal delivery have also been validated $(20,53)$. The identification of the compounds using the mass spectral library must be interpreted with caution as they have not been confirmed using analytical standards. And finally, only DC ES was used; several different waveforms and delivery methods of ES are available in wound management $(38,60)$. 
In conclusion, we have successfully utilised a human cutaneous wound biofilm model to assess VOCs as potential biomarkers and used ES as an alternative to anti-microbial treatment, showing the potential of both for chronic wound care. This study provides a platform for future work investigating the use of VOC biomarkers as diagnostic and monitoring tools in wound infections which could ultimately lead to improved clinical outcomes. 


\section{Acknowledgements}

The Bioimaging Facility microscopes used in this study were purchased with grants from BBSRC, Wellcome and the University of Manchester Strategic Fund. A special thank you goes to Roger Meadows for help with the microscopy. The authors wish to thank Helen Toogood for her invaluable assistance in using the GCMS facilities. The authors wish to thank Philip Foden for statistical advice.

Source of funding: None

Conflict of interest: The authors state no conflict of interest. 


\section{Abbreviations}

CFU- Colony-forming unit

DC - Direct current

ES - Electrical stimulation

FISH - Fluorescence in situ hybridisation

GCMS - gas chromatography-mass spectrometry

MSSA - Methicillin sensitive Staphylococcus aureus

PA - Pseudomonas aeruginosa

SSI - Surgical site infection

VOC - volatile organic compound 


\section{References}

1. James GA, Swogger E, Wolcott R, Pulcini E, Secor P, Sestrich J, et al. Biofilms in chronic wounds. Wound Repair Regen 2008;16(1):37-44.

2. Edwards R, Harding KG. Bacteria and wound healing. Curr Opin Infect Dis 2004;17(2):91-6.

3. Jones CE, Kennedy JP. Treatment Options to Manage Wound Biofilm. Adv Wound Care (New Rochelle) 2012;1(3):120-6.

4. Davies J. Microbes have the last word. A drastic re-evaluation of antimicrobial treatment is needed to overcome the threat of antibiotic-resistant bacteria. EMBO Rep 2007;8(7):616-21.

5. Gardner SE, Frantz RA, Doebbeling BN. The validity of the clinical signs and symptoms used to identify localized chronic wound infection. Wound Repair Regen $2001 ; 9(3): 178-86$.

6. Bowler PG, Duerden BI, Armstrong DG. Wound microbiology and associated approaches to wound management. Clin Microbiol Rev 2001;14(2):244-69.

7. Ganesh K, Sinha M, Mathew-Steiner SS, Das A, Roy S, Sen CK. Chronic Wound Biofilm Model. Adv Wound Care (New Rochelle) 2015;4(7):382-8.

8. Sethi S, Nanda R, Chakraborty T. Clinical application of volatile organic compound analysis for detecting infectious diseases. Clin Microbiol Rev 2013;26(3):462-75.

9. Lourenço C, Turner C. Breath analysis in disease diagnosis: methodological considerations and applications. Metabolites 2014;4(2):465-98.

10. Boots AW, van Berkel JJ, Dallinga JW, Smolinska A, Wouters EF, van Schooten FJ. The versatile use of exhaled volatile organic compounds in human health and disease. $J$ Breath Res 2012;6(2):027108. 
11. Ud-Din S, Bayat A. Electrical stimulation and cutaneous wound healing: a review of clinical evidence. 2014;2:445-67.

12. Cole W. Human Acellular Dermal Matrix Paired With Silver-zinc Coupled Electroceutical Dressing Results in Rapid Healing of Complicated Diabetic Wounds of Mixed Etiology: A Novel Case Series. Wounds 2016;28(7):241-7.

13. Nair HKR. Microcurrent as an adjunct therapy to accelerate chronic wound healing and reduce patient pain. $J$ Wound Care 2018;27(5):296-306.

14. Khouri C, Kotzki S, Roustit M, Blaise S, Gueyffier F, Cracowski JL. Hierarchical evaluation of electrical stimulation protocols for chronic wound healing: An effect size metaanalysis. Wound Repair Regen 2017;25(5):883-91.

15. Zhang L, Weng C, Zhao Z, Fu X. Extracorporeal shock wave therapy for chronic wounds: A systematic review and meta-analysis of randomized controlled trials. Wound Repair Regen 2017;25(4):697-706.

16. Banerjee J, Das Ghatak P, Roy S, Khanna S, Hemann C, Deng B, et al. Silver-zinc redox-coupled electroceutical wound dressing disrupts bacterial biofilm. PLoS One 2015;10(3):e0119531.

17. Maijer A, Gessner A, Trumpatori B, Varhus JD. Bioelectric Dressing Supports Complex Wound Healing in Small Animal Patients. Top Companion Anim Med $2018 ; 33(1): 21-8$

18. Watters C, Kay M. Eradication of Wound Biofilms by Electrical Stimulation. In: Rumbaugh KP, Ahmad I, editors. Antibiofilm Agents: From Diagnosis to Treatment and Prevention. Berlin, Heidelberg: Springer Berlin Heidelberg, 2014:425-47.

19. Nair SV, Baranwal G, Chatterjee M, Sachu A, Vasudevan AK, Bose C, et al. Antimicrobial activity of plumbagin, a naturally occurring naphthoquinone from Plumbago 
rosea, against Staphylococcus aureus and Candida albicans. Int J Med Microbiol 2016;306(4):237-48.

20. Yang Q, Phillips PL, Sampson EM, Progulske-Fox A, Jin S, Antonelli P, et al. Development of a novel ex vivo porcine skin explant model for the assessment of mature bacterial biofilms. Wound Repair Regen 2013;21(5):704-14.

21. Thet NT, Alves DR, Bean JE, Booth S, Nzakizwanayo J, Young AE, et al. Prototype Development of the Intelligent Hydrogel Wound Dressing and Its Efficacy in the Detection of Model Pathogenic Wound Biofilms. ACS Appl Mater Interfaces 2016;8(24):14909-19.

22. Alhusein N, Blagbrough IS, Beeton ML, Bolhuis A, De Bank PA. Electrospun Zein/PCL Fibrous Matrices Release Tetracycline in a Controlled Manner, Killing Staphylococcus aureus Both in Biofilms and Ex Vivo on Pig Skin, and are Compatible with Human Skin Cells. Pharm Res 2016;33(1):237-46.

23. Phillips PL, Yang Q, Schultz GS. The effect of negative pressure wound therapy with periodic instillation using antimicrobial solutions on Pseudomonas aeruginosa biofilm on porcine skin explants. Int Wound J 2013;10 Suppl 1:48-55.

24. Sullivan TP, Eaglstein WH, Davis SC, Mertz P. The pig as a model for human wound healing. Wound Repair Regen 2001;9(2):66-76.

25. Ashrafi M, Novak-Frazer L, Bates M, Baguneid M, Alonso-Rasgado T, RautemaaRichardson R, et al. 621 Novel diagnostic approach in detecting skin infection: Identification of bacterial-specific volatile organic compounds in bacterial biofilms on human cutaneous wound models. Journal of Investigative Dermatology 2017;137(5, Supplement 1):S107.

26. Nieminen MT, Novak-Frazer L, Rautemaa W, Rajendran R, Sorsa T, Ramage G, et al. A novel antifungal is active against Candida albicans biofilms and inhibits mutagenic acetaldehyde production in vitro. PLoS One 2014;9(7):e101859. 
27. Mendoza-Garcia J, Sebastian A, Alonso-Rasgado T, Bayat A. Ex vivo evaluation of the effect of photodynamic therapy on skin scars and striae distensae. Photodermatol Photoimmunol Photomed 2015;31(5):239-51.

28. Sebastian A, Iqbal SA, Colthurst J, Volk SW, Bayat A. Electrical stimulation enhances epidermal proliferation in human cutaneous wounds by modulating p53-SIVA1 interaction. J Invest Dermatol 2015;135(4):1166-74.

29. Sebastian A, Syed F, McGrouther DA, Colthurst J, Paus R, Bayat A. A novel in vitro assay for electrophysiological research on human skin fibroblasts: degenerate electrical waves downregulate collagen I expression in keloid fibroblasts. Exp Dermatol 2011;20(1):64-8.

30. Nuccitelli R, Nuccitelli P, Li C, Narsing S, Pariser DM, Lui K. The electric field near human skin wounds declines with age and provides a noninvasive indicator of wound healing. Wound repair and regeneration : official publication of the Wound Healing Society [and] the European Tissue Repair Society 2011;19(5):645-55.

31. Nuccitelli R, Nuccitelli P, Ramlatchan S, Sanger R, Smith PJ. Imaging the electric field associated with mouse and human skin wounds. Wound repair and regeneration : official publication of the Wound Healing Society [and] the European Tissue Repair Society 2008;16(3):432-41.

32. Brunner M, Stabeta H, Möller JG, Schrolnberger C, Erovic B, Hollenstein U, et al. Target site concentrations of ciprofloxacin after single intravenous and oral doses. Antimicrob Agents Chemother 2002;46(12):3724-30.

33. Marshall NJ, Goodwin CJ, Holt SJ. A critical assessment of the use of microculture tetrazolium assays to measure cell growth and function. Growth Regul 1995;5(2):69-84.

34. Bland JM, Altman DG. Calculating correlation coefficients with repeated observations: Part 2--Correlation between subjects. BMJ 1995;310(6980):633. 
35. Høiby N, Bjarnsholt T, Givskov M, Molin S, Ciofu O. Antibiotic resistance of bacterial biofilms. Int J Antimicrob Agents 2010;35(4):322-32.

36. Messerli MA, Graham DM. Extracellular electrical fields direct wound healing and regeneration. The Biological bulletin 2011;221(1):79-92.

37. Hong SH, Jeong J, Shim S, Kang H, Kwon S, Ahn KH, et al. Effect of electric currents on bacterial detachment and inactivation. Biotechnol Bioeng 2008;100(2):379-86.

38. Ashrafi M, Alonso-Rasgado T, Baguneid M, Bayat A. The efficacy of electrical stimulation in lower extremity cutaneous wound healing: A systematic review. Exp Dermatol 2017;26(2):171-8

39. Ehrensberger MT, Tobias ME, Nodzo SR, Hansen LA, Luke-Marshall NR, Cole RF, et al. Cathodic voltage-controlled electrical stimulation of titanium implants as treatment for methicillin-resistant Staphylococcus aureus periprosthetic infections. Biomaterials 2015;41:97-105.

40. van der Borden AJ, Maathuis PG, Engels E, Rakhorst G, van der Mei HC, Busscher HJ, et al. Prevention of pin tract infection in external stainless steel fixator frames using electric current in a goat model. Biomaterials 2007;28(12):2122-6.

41. Del Pozo JL, Rouse MS, Euba G, Kang CI, Mandrekar JN, Steckelberg JM, et al. The electricidal effect is active in an experimental model of Staphylococcus epidermidis chronic foreign body osteomyelitis. Antimicrob Agents Chemother 2009;53(10):4064-8.

42. Paryavi E, Yanko M, Jaffe D, Nimmgadda N, Nouveau J, Schiavone J, et al. Implantable direct current spinal fusion stimulators do not decrease implant-related infections in a rabbit model. Am J Orthop (Belle Mead NJ) 2014;43(5):E98-E104.

43. Gilotra M, Griffith C, Schiavone J, Nimmagadda N, Noveau J, Ludwig SC. Capacitive coupling reduces instrumentation-related infection in rabbit spines: a pilot study. Clin Orthop Relat Res 2012;470(6):1646-51. 
44. Nodzo SR, Tobias M, Ahn R, Hansen L, Luke-Marshall NR, Howard C, et al. Cathodic Voltage-controlled Electrical Stimulation Plus Prolonged Vancomycin Reduce Bacterial Burden of a Titanium Implant-associated Infection in a Rodent Model. Clin Orthop Relat Res 2016;474(7):1668-75.

45. Ashrafi M, Baguneid M, Alonso-Rasgado T, Rautemaa-Richardson R, Bayat A. Cutaneous wound biofilm and the potential for electrical stimulation in management of the microbiome. Future Microbiol 2017;12:337-57.

46. Haddad PA, Mah TF, Mussivand T. In Vitro Assessment of Electric Currents Increasing the Effectiveness of Vancomycin Against Staphylococcus epidermidis Biofilms. Artif Organs 2016;40(8):804-10.

47. Zhang J, Neoh KG, Hu X, Kang ET. Mechanistic insights into response of Staphylococcus aureus to bioelectric effect on polypyrrole/chitosan film. Biomaterials 2014;35(27):7690-8.

48. del Pozo JL, Rouse MS, Mandrekar JN, Sampedro MF, Steckelberg JM, Patel R. Effect of electrical current on the activities of antimicrobial agents against Pseudomonas aeruginosa, Staphylococcus aureus, and Staphylococcus epidermidis biofilms. Antimicrob Agents Chemother 2009;53(1):35-40.

49. Jass J, Lappin-Scott HM. The efficacy of antibiotics enhanced by electrical currents against Pseudomonas aeruginosa biofilms. J Antimicrob Chemother 1996;38(6):987-1000.

50. Barker AT, Jaffe LF, Vanable JW. The glabrous epidermis of cavies contains a powerful battery. Am J Physiol 1982;242(3):R358-66.

51. Wolcott RD, Rumbaugh KP, James G, Schultz G, Phillips P, Yang Q, et al. Biofilm maturity studies indicate sharp debridement opens a time- dependent therapeutic window. $J$ Wound Care 2010;19(8):320-8. 
52. Raz-Pasteur A, Ullmann Y, Berdicevsky I. The pathogenesis of Candida infections in a human skin model: scanning electron microscope observations. ISRN Dermatol 2011;2011:150642.

53. Schaudinn C, Dittmann C, Jurisch J, Laue M, Günday-Türeli N, Blume-Peytavi U, et al. Development, standardization and testing of a bacterial wound infection model based on ex vivo human skin. PLoS One 2017;12(11):e0186946.

54. Healy B, Freedman A. Infections. BMJ 2006;332(7545):838-41.

55. Ashrafi M, Bates M, Baguneid M, Alonso-Rasgado T, Rautemaa-Richardson R, Bayat A. Volatile organic compound detection as a potential means of diagnosing cutaneous wound infections. Wound Repair Regen 2017.

56. Wilson AD, Baietto M. Advances in electronic-nose technologies developed for biomedical applications. Sensors (Basel) 2011;11(1):1105-76.

57. Nardi-Agmon I, Abud-Hawa M, Liran O, Gai-Mor N, Ilouze M, Onn A, et al. Exhaled Breath Analysis for Monitoring Response to Treatment in Advanced Lung Cancer. $J$ Thorac Oncol 2016;11(6):827-37.

58. Greenwood JE, Crawley BA, Clark SL, Chadwick PR, Ellison DA, Oppenheim BA, et al. Monitoring wound healing by odour. J Wound Care 1997;6(5):219-21 .

59. Ashrafi M, Novak-Frazer L, Bates M, Baguneid M, Alonso-Rasgado T, Xia G, et al. Validation of biofilm formation on human skin wound models and demonstration of clinically translatable bacteria-specific volatile signatures. Sci Rep 2018;8(1):9431.

60. Ashrafi M, Alonso-Rasgado T, Baguneid M, Bayat A. The efficacy of electrical stimulation in experimentally induced cutaneous wounds in animals. Vet Dermatol 2016;27(4):235-e57. 


\section{Figure legends}

Figure 1. Study design. Biofilm appearance, viability, metabolic activity and biomass were evaluated in vitro and ex vivo at multiple time points using a number of techniques to determine the effects of antibiotics, ES and combination treatment. VOCs were identified and their relative abundance compared to determine their use as predictors of infection and to monitor treatment. VOCs, volatile organic compounds; ES, electrical stimulation.

Figure 2. Visualisation and comparison of viability, metabolic activity and biomass of in vitro MSSA biofilms following exposure to antibiotics or ES. Biofilm viability was determined by (A) CFU assessment or (B) SYTO ${ }^{\circledR} 9$ quantification using Definiens Tissue Studio software. Metabolic activity was determined by the XTT reduction assay with $(\mathbf{C})$ the substrate (i.e. coverslip) in situ or (D) the biofilm separated from the substrate. (E) Biomass was determined by Quant-iT PicoGreen dsDNA reagent assay. Mean $\pm 95 \%$ confidence intervals $(\mathrm{n}=6), * \mathrm{P}<0.05, * * \mathrm{P}<0.01$, *** $\mathrm{P}<0.001$, as determined by generalised

estimating equation model with accompanying Bonferroni post hoc analyses. Means were compared at each time point to the control treatment. (F) LIVE/DEAD staining. Viable bacteria are stained green with the nucleic acid stain $\mathrm{SYTO}^{\circledR} 9$ and extracellular DNA or dead bacteria are stained red with the nucleic acid stain propidium iodide. Scale bar: $10 \mu \mathrm{m}$. MSSA, methicillin sensitive Staphylococcus aureus; ES, electrical stimulation.

Figure 3. Visualisation and comparison of viability, metabolic activity and biomass of in vitro PA biofilms following exposure to antibiotics or ES. Biofilm viability was determined by (A) CFU assessment or (B) SYTO ${ }^{\circledR} 9$ quantification using Definiens Tissue Studio 
software. Metabolic activity was determined by the XTT reduction assay with (C) the substrate (i.e. coverslip) in situ or (D) the biofilm separated from substrate. (E) Biomass was determined by Quant-iT PicoGreen dsDNA reagent assay. Mean $\pm 95 \%$ confidence intervals $(\mathrm{n}=6), * \mathrm{P}<0.05, * * \mathrm{P}<0.01, * * * \mathrm{P}<0.001$, as determined by generalised estimating equation model with accompanying Bonferroni post hoc analyses. Means were compared at each time point to the control treatment. (F) LIVE/DEAD staining. Viable bacteria are stained green with the nucleic acid stain SYTO ${ }^{\circledR} 9$ and extracellular DNA or dead bacteria are stained red with the nucleic acid stain propidium iodide. Scale bar: $10 \mu \mathrm{m}$. PA, Pseudomonas aeruginosa; ES, electrical stimulation.

Figure 4. Comparison of viability, metabolic activity and biomass of ex vivo PA biofilms following exposure to ES, combined ES and antibiotic or no treatment (controls). Biofilm viability was determined by (A) CFU assessment. (B) Metabolic activity was determined by the XTT reduction assay. (C) Biomass was determined by Quant-iT PicoGreen dsDNA reagent assay. Mean $\pm 95 \%$ confidence intervals $(\mathrm{n}=12), * \mathrm{P}<0.05, * * \mathrm{P}<0.01$, *** $\mathrm{P}<0.001$, as determined by generalised estimating equation model with accompanying Bonferroni post hoc analyses. Means were compared at each time point to the control treatment. PA, Pseudomonas aeruginosa; ES, electrical stimulation.

Figure 5. Visualisation of ex vivo PA biofilms following exposure to antibiotic, ES, combined ES and antibiotic, or no treatment (controls). Microscopy of haematoxylin- and eosin-stained sections at 50x (A), 600x (B) and 1000x (C) magnification. Wound tissue is stained pink and biofilm material blue or purple. Microscopy of Gram-Twort stained sections at 600x (D) and 1000x (E) magnification. Wound tissue is stained green and biofilm material 
pink. (F) DAPI and Concanavalin A staining: viable bacteria and host cells are stained blue with the nucleic acid stain DAPI and extracellular matrix is stained red with Concanavalin A conjugate. (G) Biofilms are stained with the UniBac PNA FISH® probe. a: epidermis; b: dermis. Black scale bar: $200 \mu \mathrm{m}$. White Scale bar: $10 \mu \mathrm{m}$. PA, Pseudomonas aeruginosa; ES, electrical stimulation; DAPI, 4',6-diamidino-2-phenylindole; FISH, fluorescence in situ hybridisation.

Figure 6. Comparison of VOCs and Ln (relative abundances) from infected biofilms following exposure to antibiotic, ES, combined ES and antibiotic or no treatment (controls). A: MSSA Day 1 in vitro. B: MSSA Day 3 in vitro. C: PA Day 1 in vitro. D: PA Day 3 in vitro. (E) PA Day 3 ex vivo. Mean $\pm 95 \%$ confidence intervals $(\mathrm{n}=6)$, * $\mathrm{P}<0.05$, ** $\mathrm{P}<0.01, * * * \mathrm{P}<0.001$, as determined by generalised estimating equation model with accompanying Bonferroni post hoc analyses. Means were compared at each time point to the control treatment. +: denotes zero value. ES, electrical stimulation; VOCs, volatile organic compound; MSSA, methicillin sensitive Staphylococcus aureus; PA, Pseudomonas aeruginosa.

Figure 7. Correlations in vitro. Significant correlations between 2-methyl-1-propanol and the number of MSSA viable bacteria as determined by (A) CFU assessment; (B) SYTO ${ }^{\circledR} 9$ quantification using Definiens Tissue Studio software. (C) MSSA biofilm biomass as determined by Quant-iT PicoGreen dsDNA reagent assay; and (D) MSSA biofilm metabolic activity as determined by XTT reduction assay. (E) Significant correlation between 3-methyl1-butanol and PA biofilm biomass as determined by Quant-iT PicoGreen dsDNA reagent assay. Mean \pm SEM (n=6). Coloured data points represent the following: Grey - day 1 
control; Red - day 1 antibiotic; Yellow- day 1 electrical stimulation; Green - day 3 control; Blue - day 3 antibiotic; Purple -day 3 electrical stimulation. 

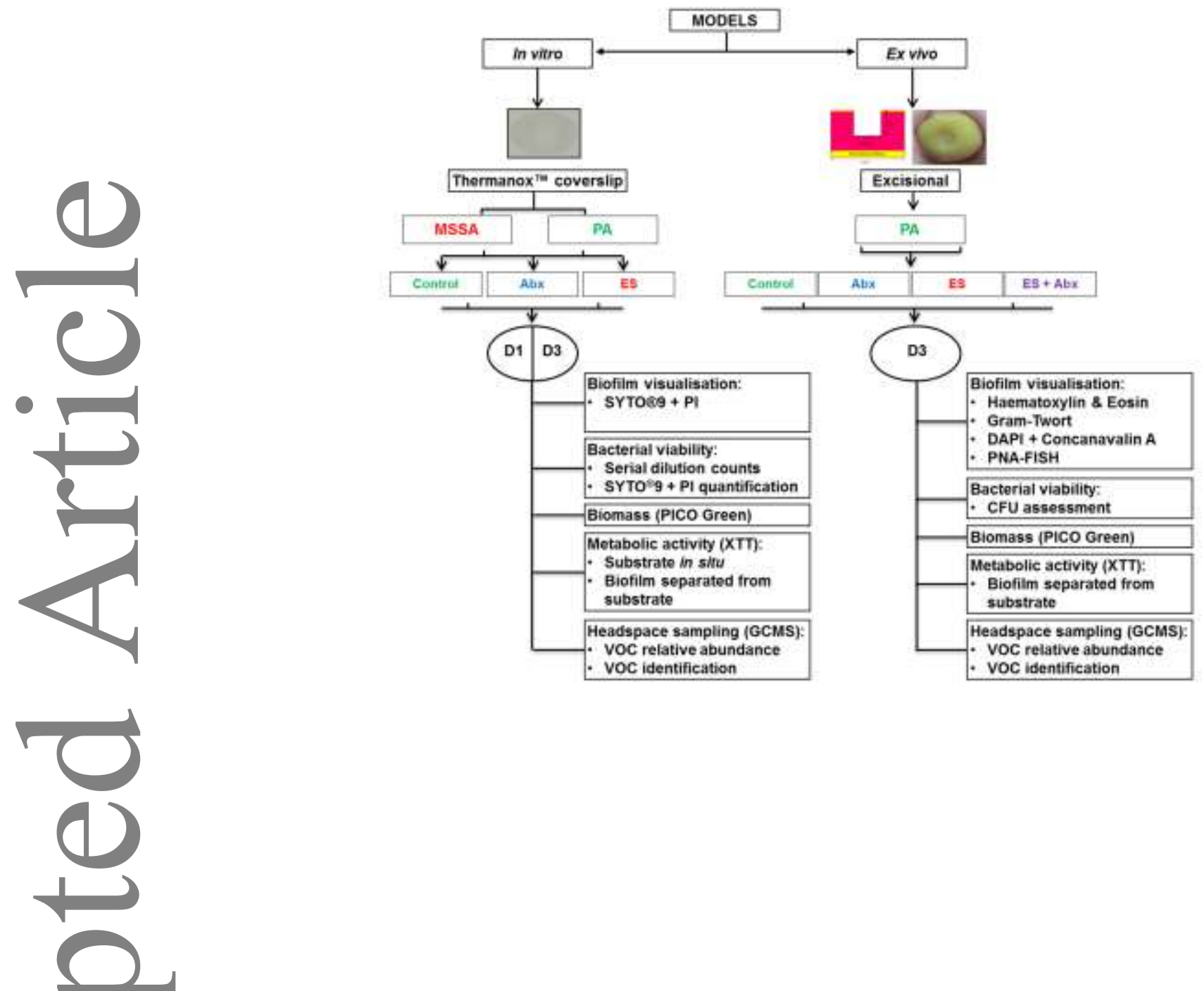

$r$

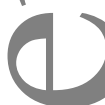

P

J

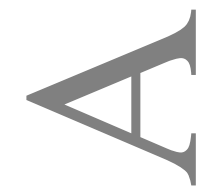

This article is protected by copyright. All rights reserved. 


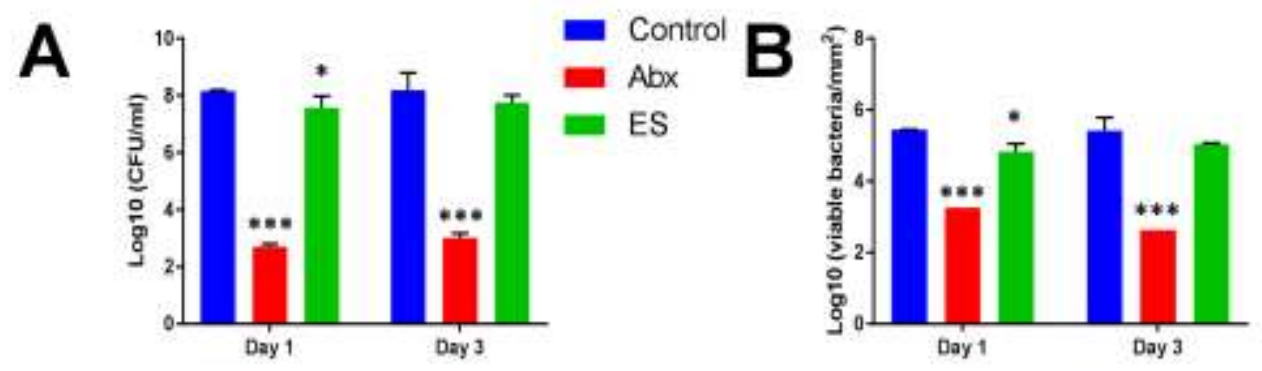

C

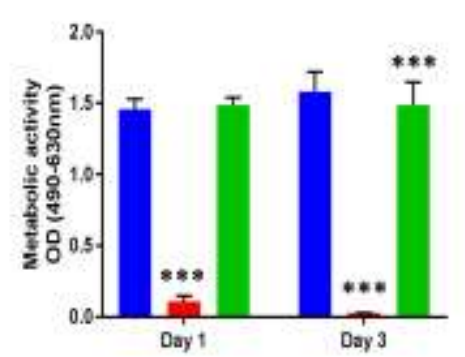

D

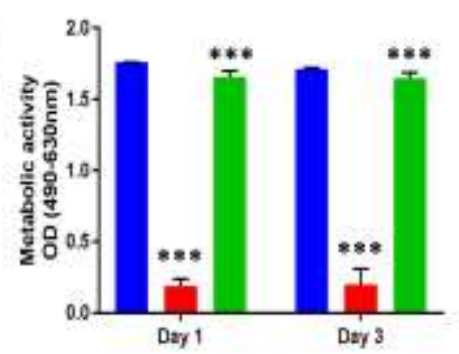

E

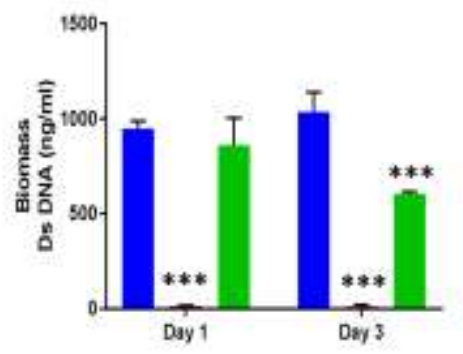

F

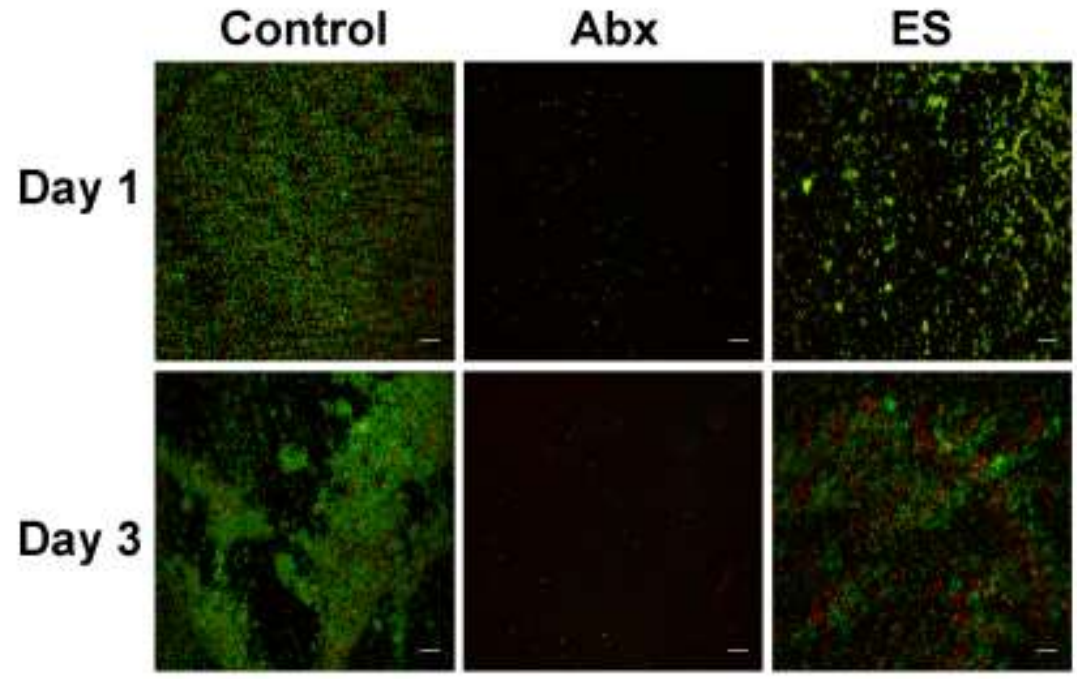




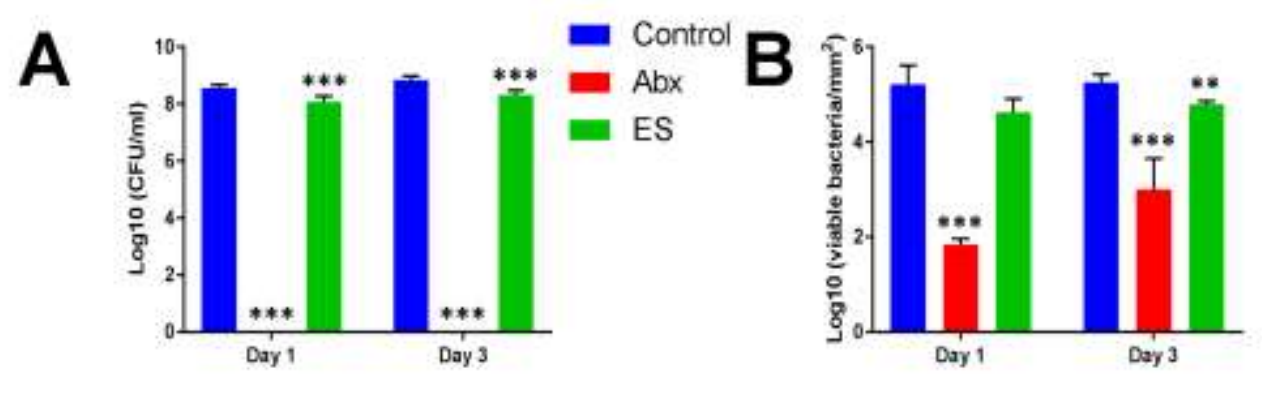

C

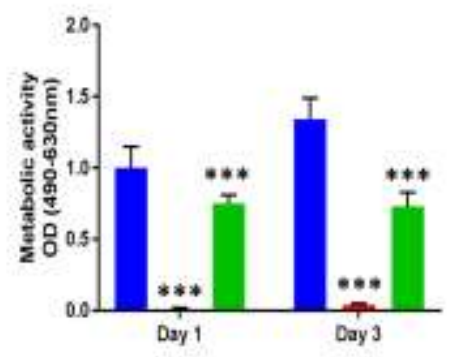

E

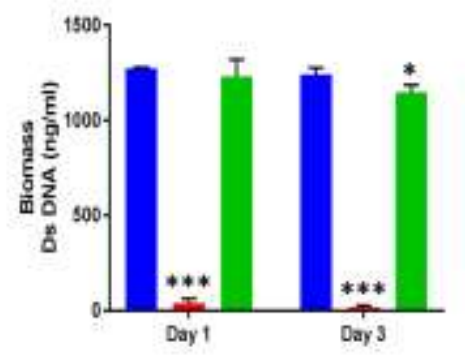

D

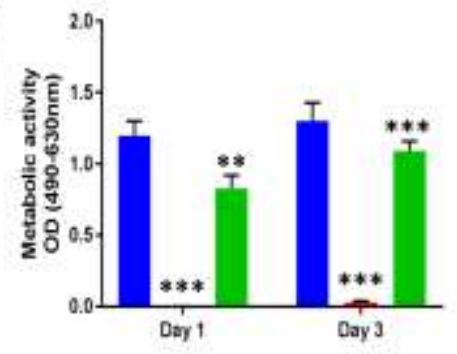

F

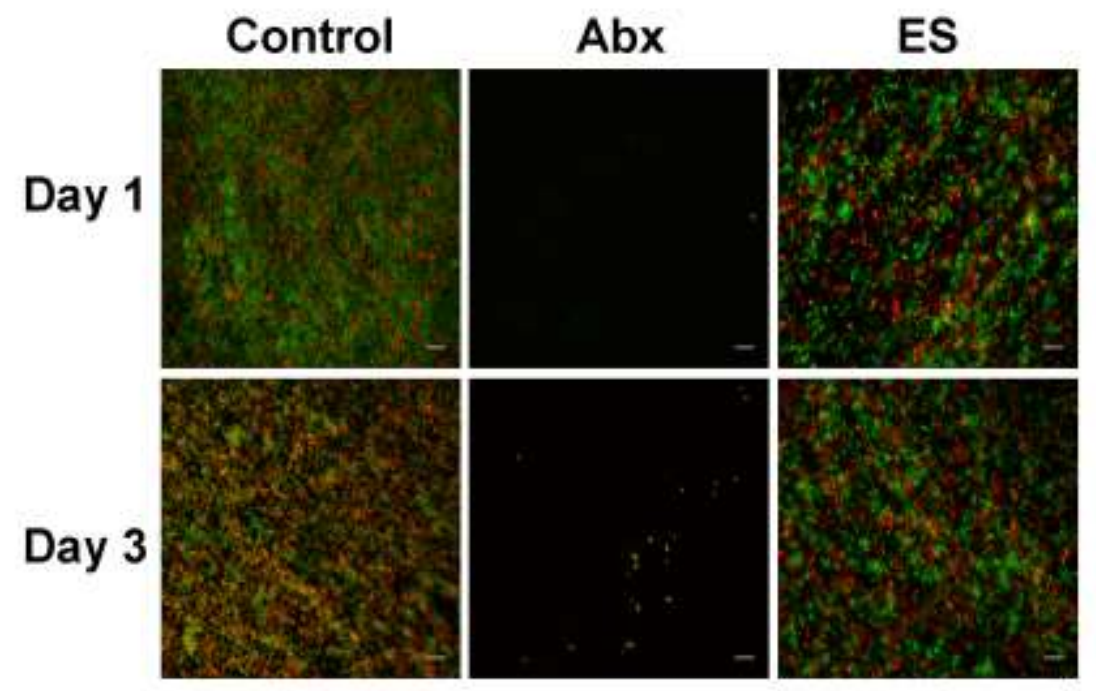



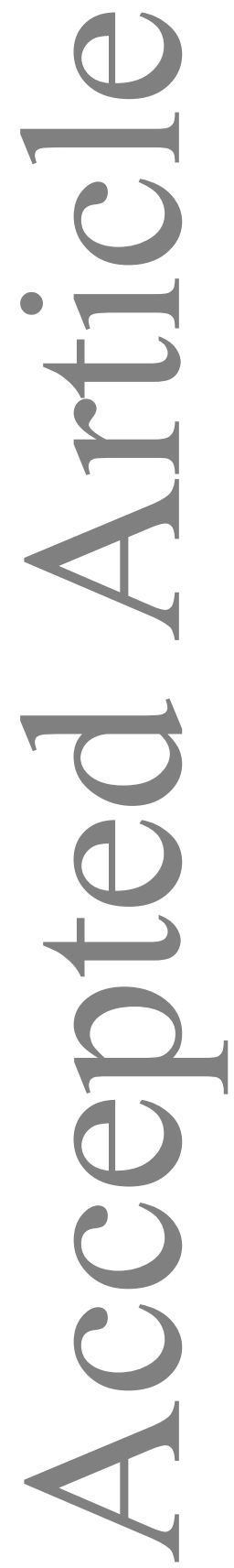

Control Abx ES ES + Abx

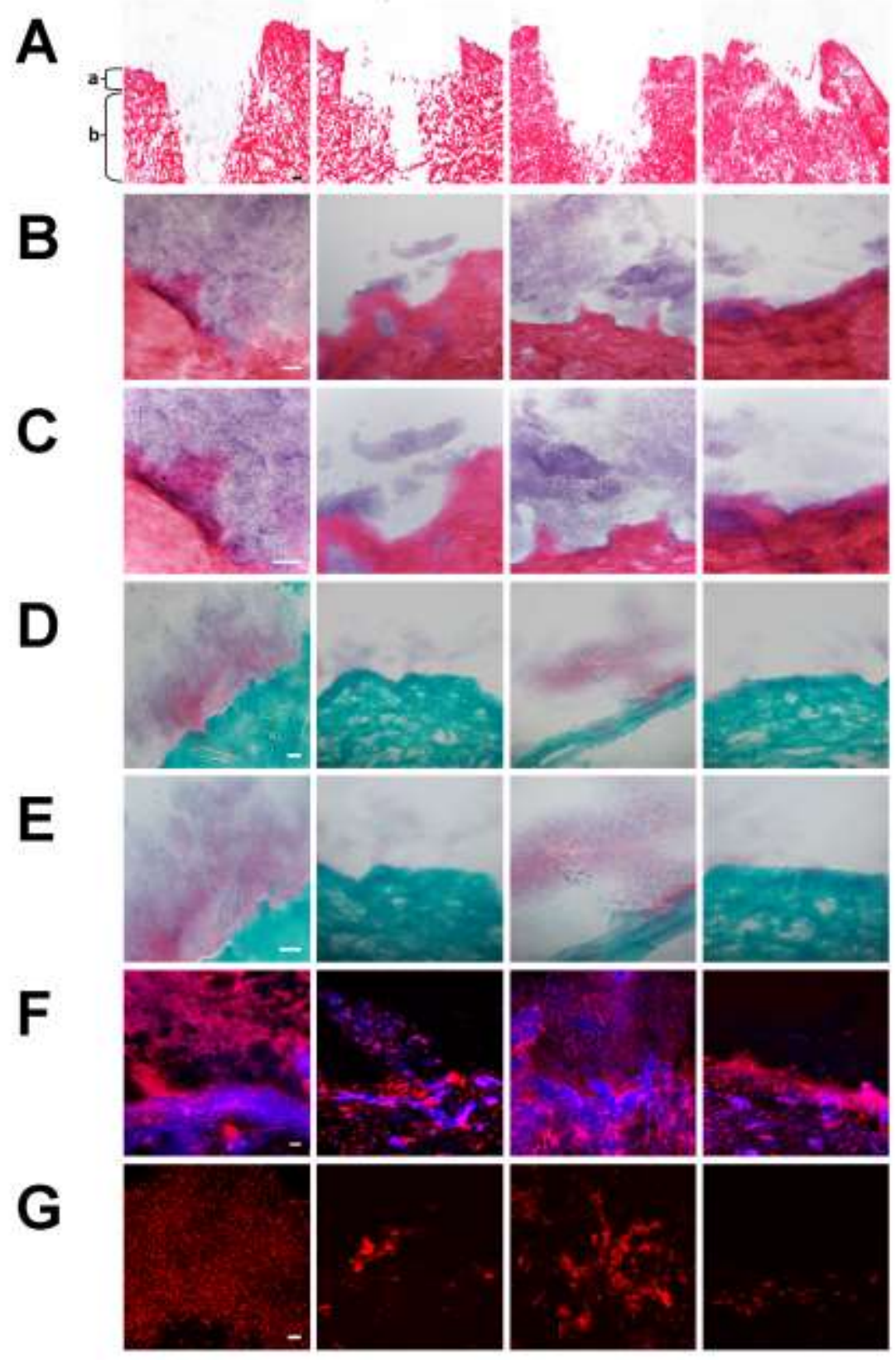


A

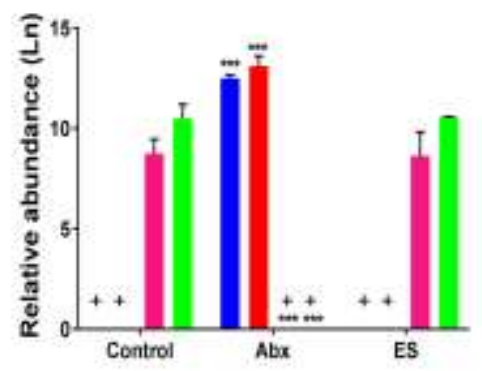

C

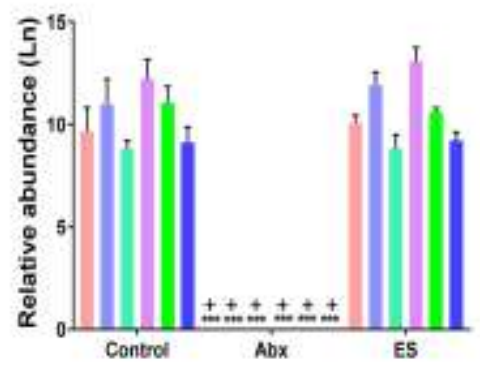

E

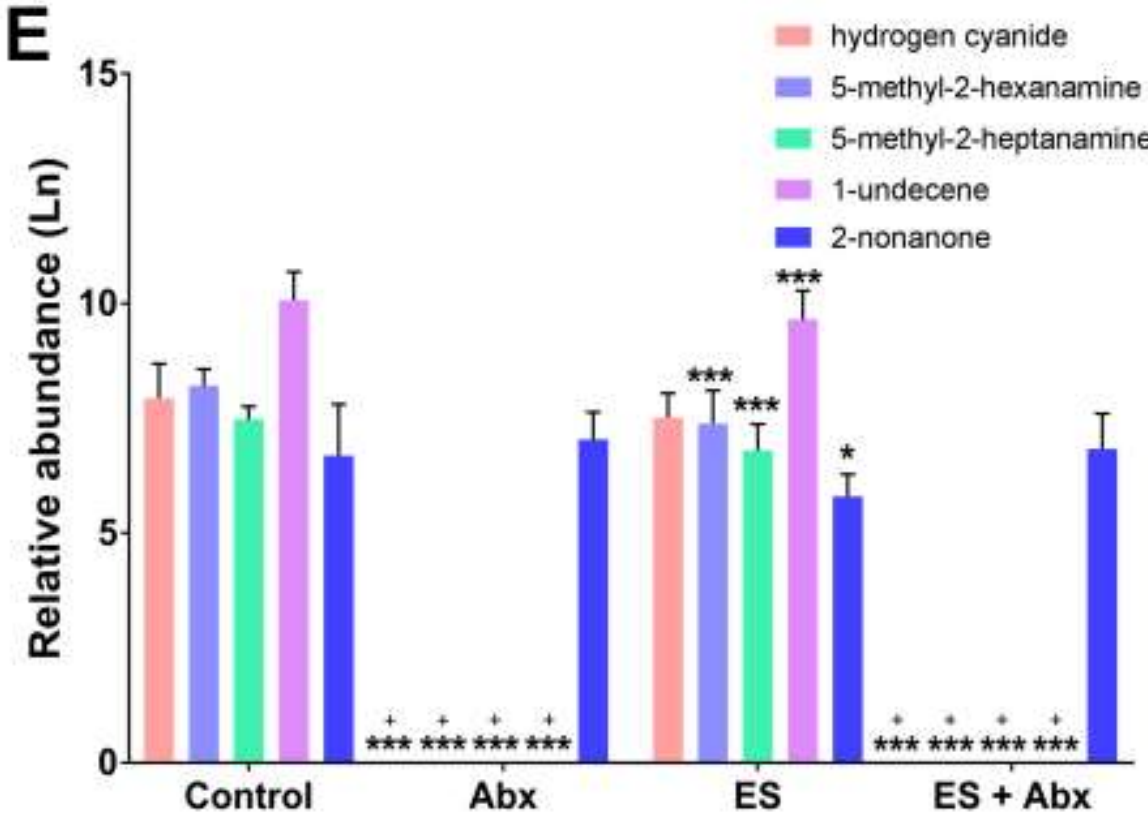

B

- Acetic acid ethenyl ester

nin 2-methyl-1-propanol

en 3-methyl-1-butanol

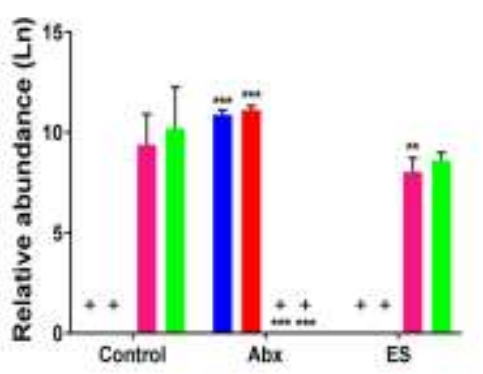

D

= hydrogen cyanide

= 5-methyl-2-hexanamine

$=5$-methyl-2-heptanamine

II- 1 -undecene

= 3-methyl-1-butanol

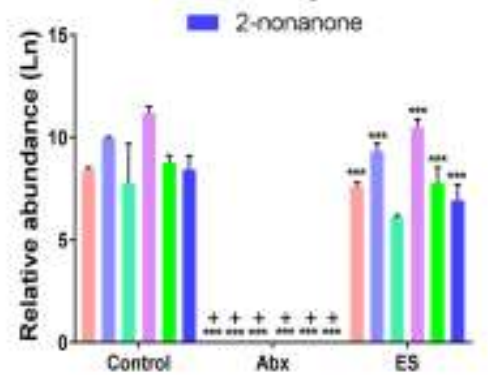

I- hydrogen cyanide

5-methyl-2-hexanamine

Abx

ES

$E S+A b x$
- Butanedione

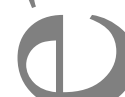

( $)$

( )

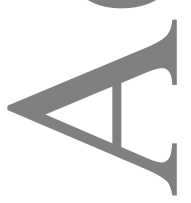




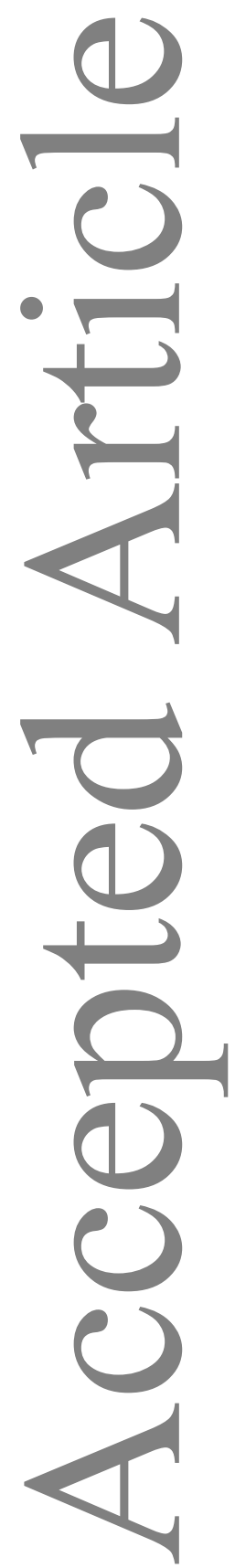




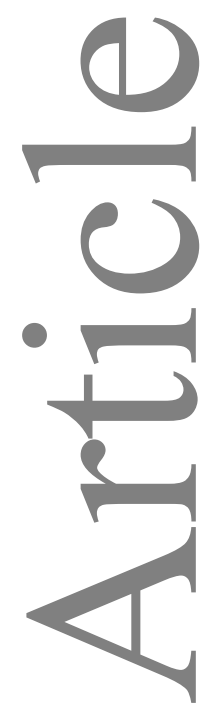

A
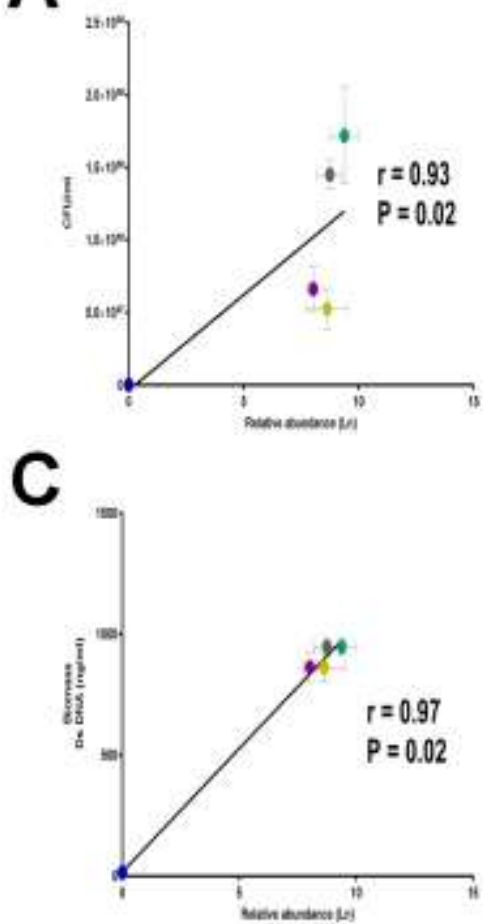

E

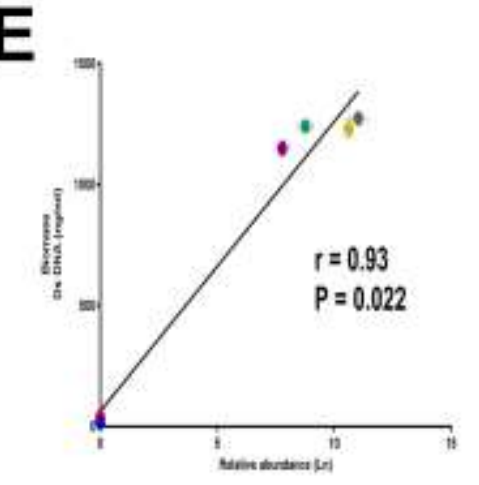

B

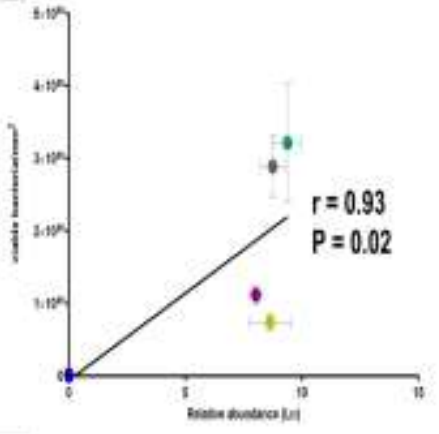

D

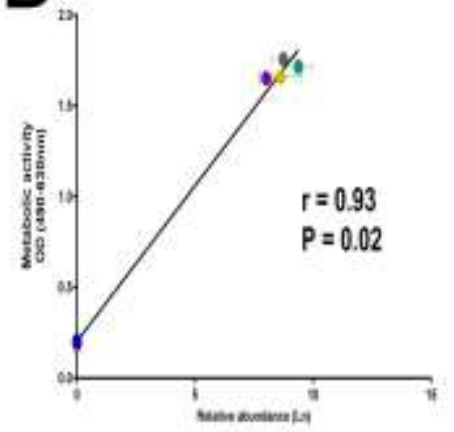

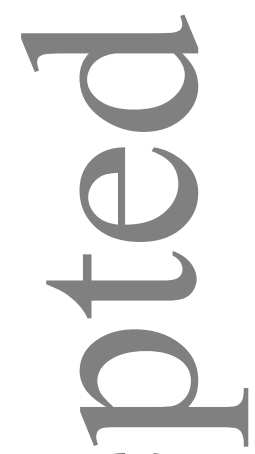

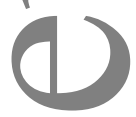

( $)$

C

$<$ 